Caso clínico

\title{
Foramen oval permeable como causa de evento vascular cerebral de presunto origen criptogénico
}

\section{Patent foramen ovale as pseudo-cryptogenic stroke}

Haro-Alcalde, Fabian Alejandro; Mendoza-Enciso, Emmanuel

Antonio; Arzate-Herrera, Alma Irina; Zavaleta-Martínez, Mariana

\section{Fabian Alejandro Haro-Alcalde}

Centenario Hospital Miguel Hidalgo, México

Emmanuel Antonio Mendoza-Enciso

Centenario Hospital Miguel Hidalgo, México

Alma Irina Arzate-Herrera

Médico General, México

Mariana Zavaleta-Martínez zavaletaalfil@gmail.com

Centenario Hospital Miguel Hidalgo, México

\section{Lux Médica}

Universidad Autónoma de Aguascalientes, México

ISSN: 2007-1655

Periodicidad: Cuatrimestral

vol. 17 , núm. 49,2022

luxmedica.editorial@gmail.com

Recepción: 08 Agosto 2021

Aprobación: 25 Noviembre 2021

URL:

https://revistas.uaa.mx/index.php/luxmedica/article/view/3317

DOI:

https://doi.org/10.33064/491m20223317

\begin{abstract}
Resumen: El foramen oval permeable es una de las causas de enfermedad vascular cerebral isquémico, el cual, de no buscarse, podría encasillarse en las entidades de origen criptogénico. Esta entidad debe buscarse como entidad causal de evento vascular cerebral en aquellas personas con causa presuntamente criptogénica, ya que de no tratarse adecuadamente podrá tener consecuencias desfavorables para el paciente.
\end{abstract}

Palabras clave: Foramen oval permeable, enfermedad vascular cerebral.

\begin{abstract}
A patent foramen ovale is an ischemic stroke cause which if not sought, could be pigeonholed into entities of cryptogenic origin. This disease should be sought as the causal entity of a cerebrovascular event in those with a presumed cryptogenic cause, since if it is not treated properly, it may have unfavorable consequences for the patient.
\end{abstract}

Keywords: Patent foramen ovale, ischemic stroke.

\section{(1) (1)}

Esta obra está bajo una Licencia Creative Commons Atribución-

NoComercial-CompartirIgual 4.0 Internacional.

\section{INTRODUCCIÓN}

El foramen oval permeable (FOP) es la cardiopatía congénita más prevalente en la población adulta, la cual ha sido reconocida históricamente como una causa de ictus isquémico de origen criptogénico en adultos jóvenes. ${ }^{4}$ Parte del abordaje incluye la realización de ecocardiografía cardiaca con la búsqueda intencionada de FOP, por ser una causa potencialmente tratable. En este caso, se ha propuesto la embolización paradójica como su principal mecanismo fisiopatológico, sin menospreciar el reto diagnóstico que implica definir si es el principal factor etiológico, o nos encontramos ante un hallazgo incidental, dada la alta prevalencia de este defecto interauricular. ${ }^{3}$ 


\section{CASO CLÍNICO}

Paciente femenino de 37 años de edad, originaria y residente de la zona urbana de Jerez, Zacatecas, casada, ama de casa. Dentro de sus antecedentes heredo-familiares, destaca únicamente que su abuela materna tiene hipertensión arterial sistémica. Como antecedentes personales no patológicos, no convive con animales, sin toxicomanías, adecuada alimentación, sin antecedente a exposición a biomasa o mielotóxicos, sin historia de prácticas de riesgo para enfermedades de transmisión sexual. Sin tatuajes ni perforaciones. Previo al inicio del padecimiento actual la paciente se refería sana.

\section{Antecedentes Gineco-Obstétricos}

Menarca a los 11 años, ritmo regular $28 \times 3$; IVSA a los 21 años, un solo compañero sexual, aparentemente sano. G4 P4 C0 A0.

Antecedentes personales patológicos

Enfermedades crónicas: no referidas.

Quirúrgicos: no referidos.

Traumatismos: no referidos.

Transfusiones sanguíneas: no referidas.

Alergias: no referidas.

\section{Padecimiento actual}

Lo inicia un día antes de su ingreso por la noche, con habla ininteligible y dificultad para movilizar la mitad derecha del cuerpo; acude a revisión al día siguiente. Se encuentra de la siguiente manera:

120/62 mmHg FC 72 lpm Fr 18 rpm Sat.O2 96\% Temp: 36.5 ${ }^{\circ} \mathrm{C}$

Alerta, apertura ocular espontánea, realiza sonidos no comprensibles, orientación no valorada, pupilas simétricas de $3 \mathrm{~mm}$ con buena respuesta a la luz, asimetría facial, desviación de la comisura labial a la izquierda, extremidad superior derecha con fuerza muscular $2 / 5$ con reflejos miotáticos aumentados; extremidad superior izquierda y extremidades inferiores con fuerza muscular $5 / 5$, reflejos miotáticos normales, respuesta plantar flexora; cuello sin plétora yugular, bocio ni adenomegalias, tórax con ruidos respiratorios normales, precordio rítmico, no S3 ni S4, abdomen blando, depresible, rebote ausente, extremidades eutróficas, sin edema, llenado capilar inmediato.

\section{Estudios de laboratorio}

- Hb 14.2 g/dl, VCM 90.8, HCM 29.3, plaquetas 376 mil, leucocitos 7,260 .

- Glucosa $91 \mathrm{mg} / \mathrm{dl}$, Urea 26, Cr 0.8, Colesterol total 230, triglicéridos 211. 
- Tiempos de coagulación: TP 12 segundos, INR 0.8, TTP 25 segundos.

- ANAs: Resultado Negativo; C3 y C4 normales

- Anticoagulante lúpico, IgG anticardiolipina, anti B2 glucoproteína1: Negativos.

- p-ANCA, c-ANCA: negativos.

Estudios de gabinete: como parte del abordaje se efectuó ultrasonido Doppler carotídeo que resultó normal, flujo adecuado, sin lesiones sugestivas de placas de ateroma.

\section{Resonancia Magnética de Encéfalo}

En difusión se observa la lesión con restricción; en secuencia HEMO, sigue siendo hiperintensa, por lo que se descarta hemorragia. En T2 y FLAIR se observa edema cerebral y T1 más contraste no refuerza. Conclusión: EVC isquémico en ramas de M4 de arteria cerebral media izquierda (figura 1).

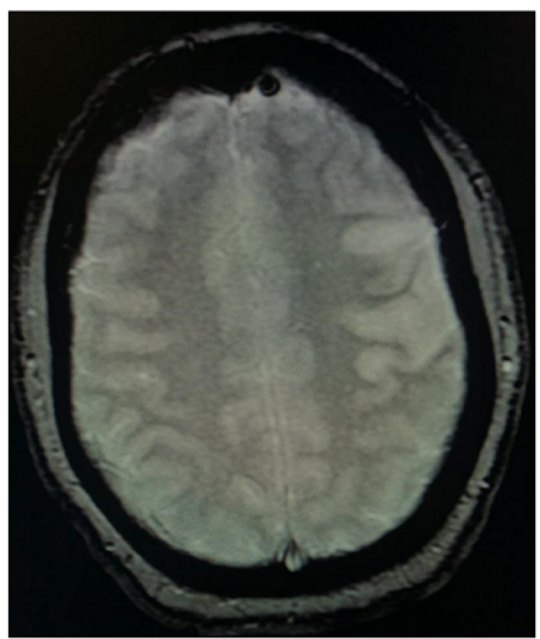

Secuencia Hemo

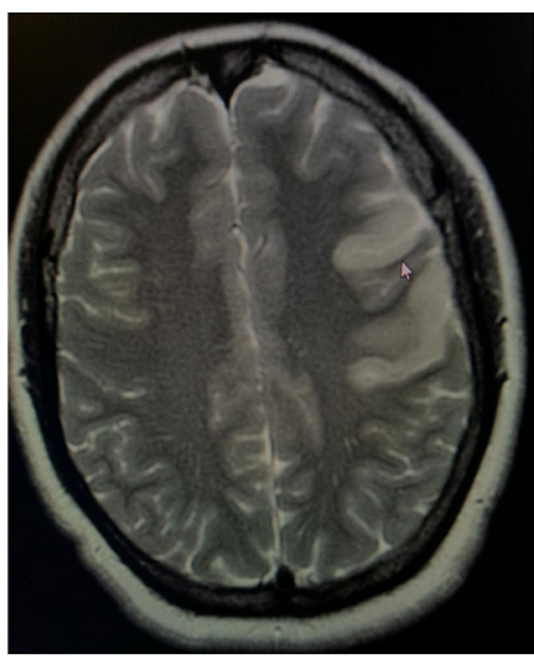

$\mathrm{T} 2$

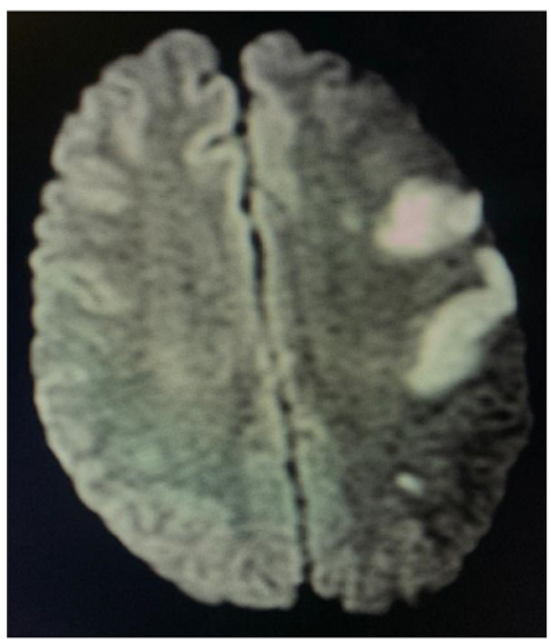

Difusión

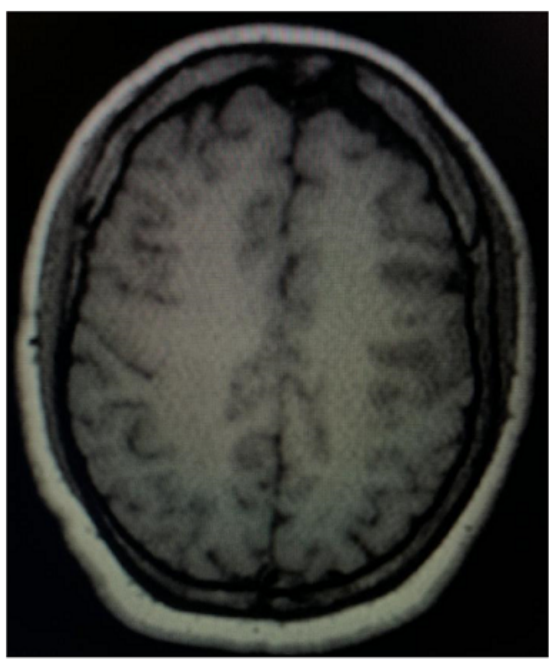

$\mathrm{T} 1$ con contraste

Figura 1. Resonancia Magnética de Encéfalo 


\section{Electrocardiograma}

Ritmo sinusal, FC $60 \mathrm{lpm}$, eje +75, PR 0.14s, QRS 0.08s, ST isoeléctrico, QTC 0.42 s (figura 2)

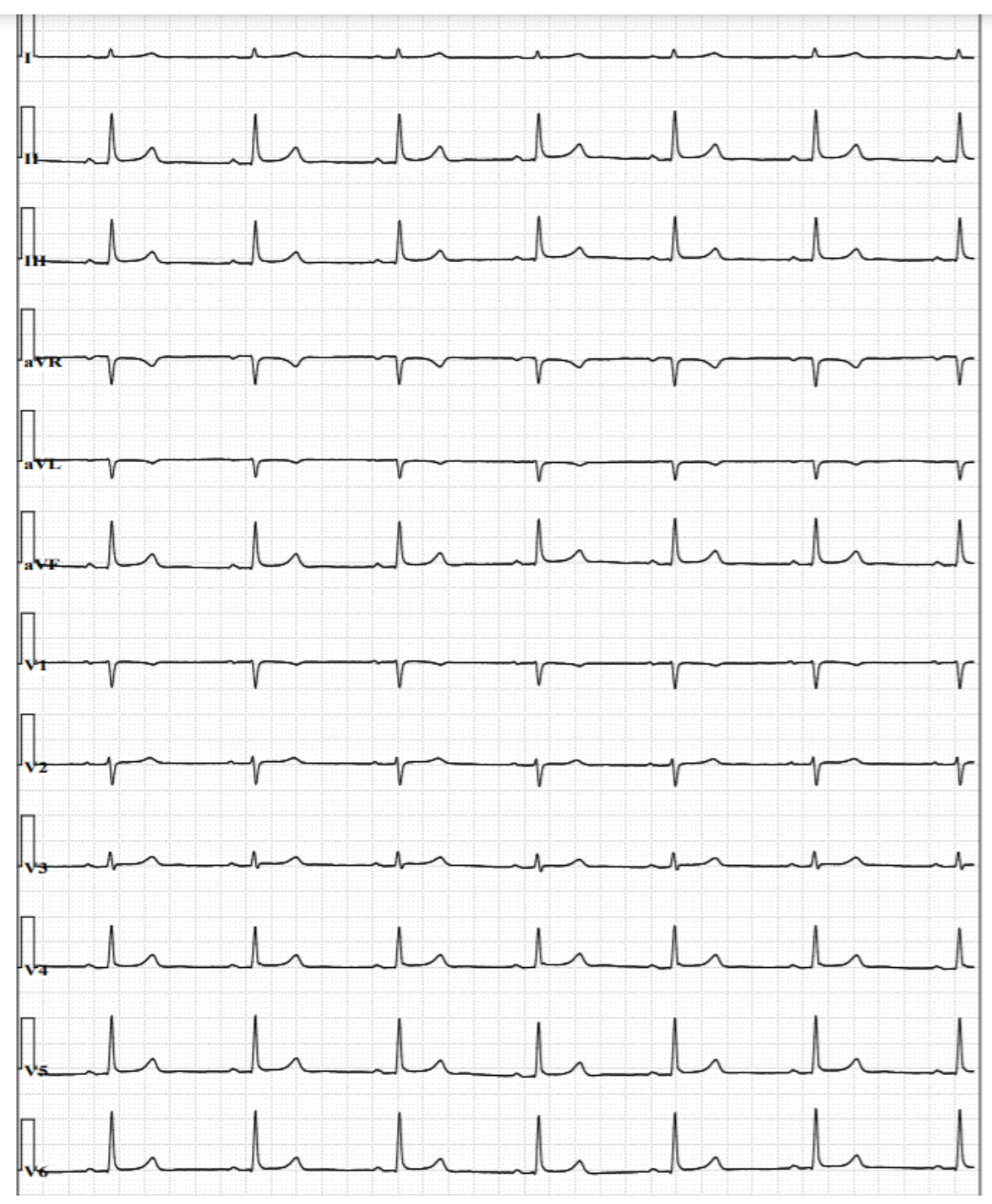

Figura 2. Electrocardiograma

Holter de 24 horas

Durante el monitoreo se mantuvo en ritmo sinusal. LA FC osciló entre 53 y $93 \mathrm{lpm}$. Presentó 585 extrasístoles ventriculares aisladas. La variabilidad de la frecuencia cardiaca fue normal (figura 3). 


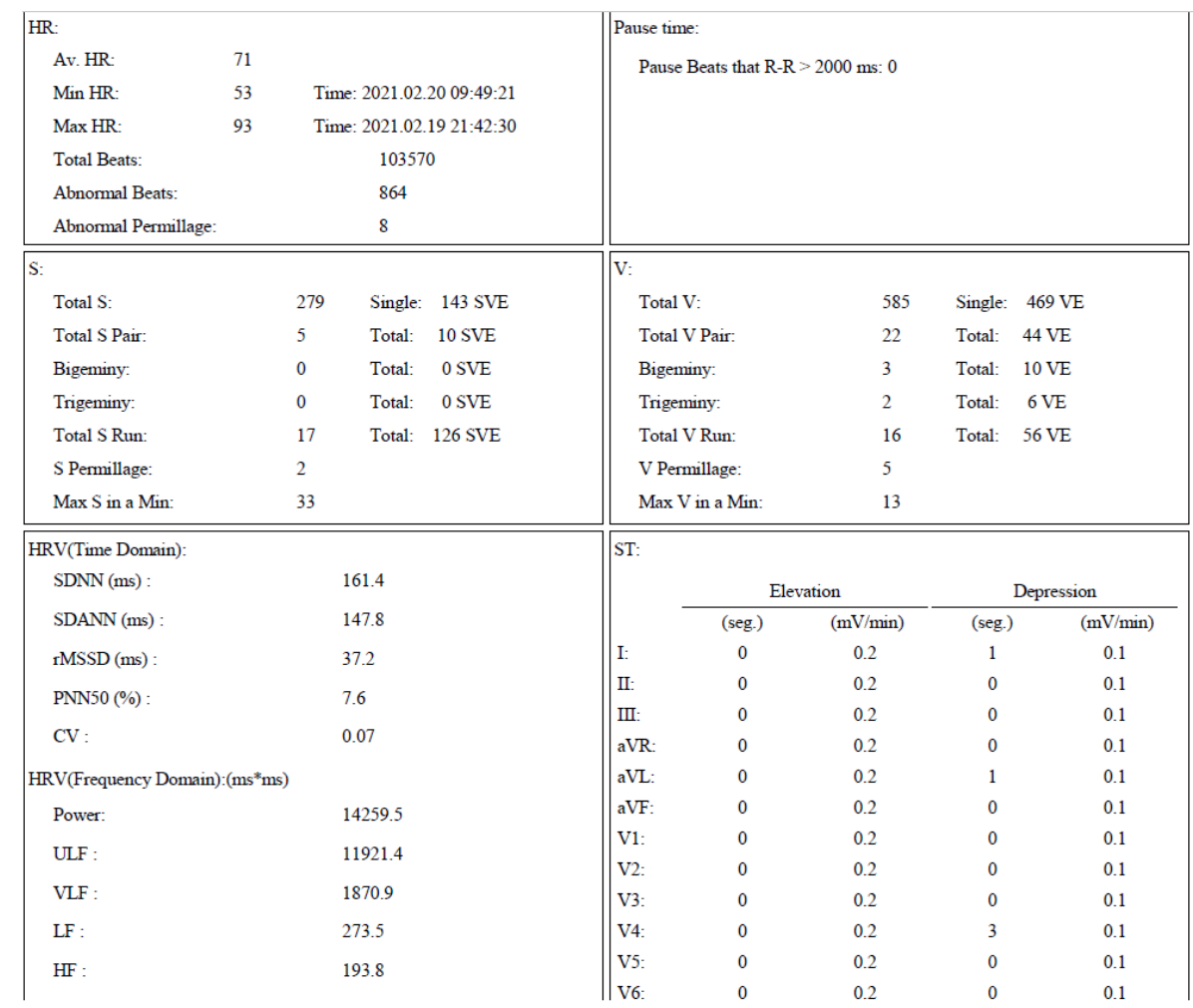

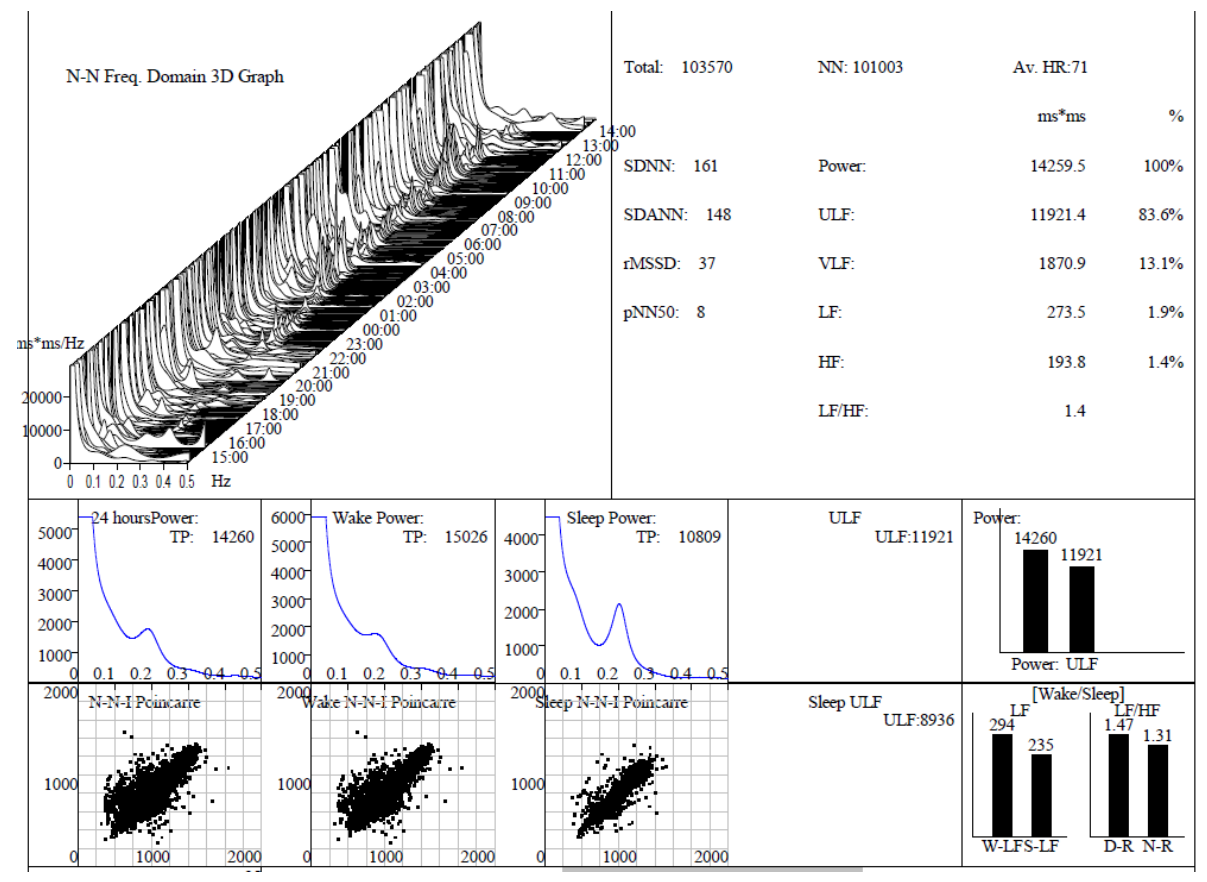

Figura 3. Holter de 24 horas 


\section{Ecocardiograma}

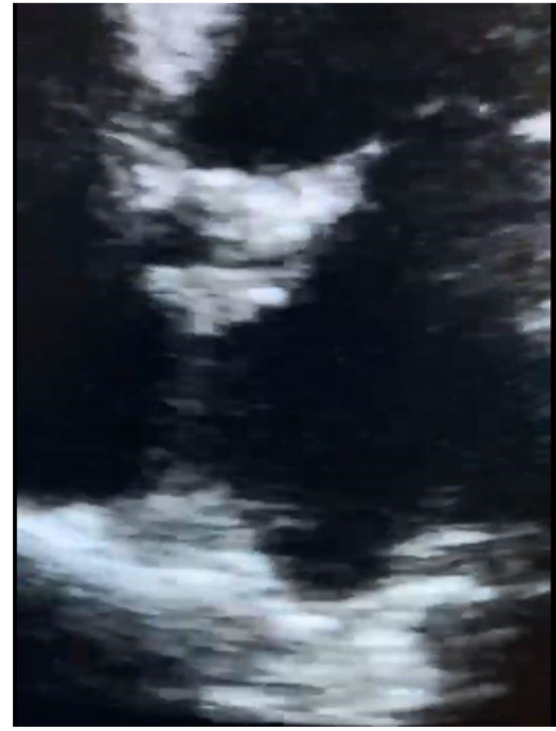

(a)

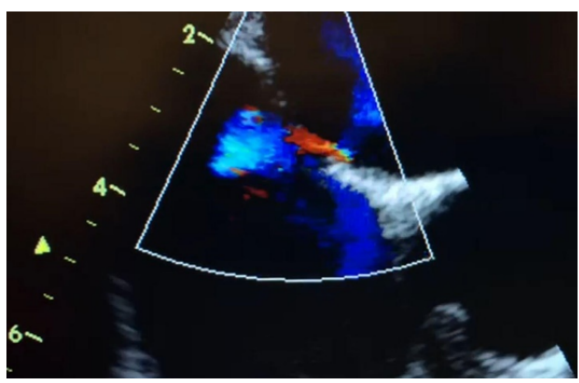

(c)

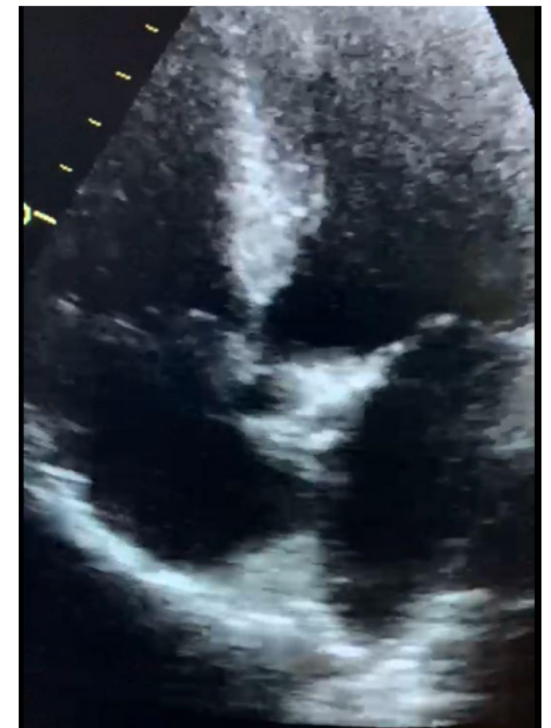

(b)

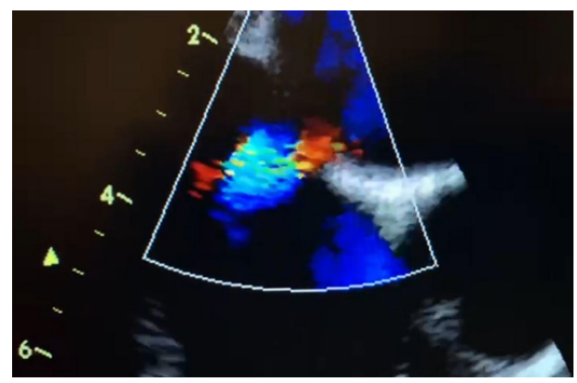

(d)

Figura 4. Ecocardiograma transtorácico

Muestra la presencia de foramen oval permeable con cortocircuito derecha-izquierda (a) y aneurisma del septum intra atrial (b). Posteriormente, con el modo Doppler y la prueba de infusión de solución salina agitada se aprecian las burbujas, de más de 30 a cavidades izquierdas en primeros tres latidos (c y d).

\section{DISCUSIÓN}

En todo el mundo, más de dos millones de adultos jóvenes tienen un accidente cerebrovascular isquémico (ACVI) cada año. ${ }^{1}$ En las últimas décadas, se ha registrado un aumento de hasta el $40 \%$ en la incidencia de ictus en adultos jóvenes.

Las posibles explicaciones para esta creciente incidencia incluyen una mayor detección con técnicas avanzadas de neuroimagen, particularmente la resonancia magnética ponderada por difusión, mayor prevalencia de factores de riesgo tradicionales modificables y aumento del consumo de drogas ilícitas y recreativas. $^{2}$

El FOP permanece presente en un $25 \%$ en la población adulta, ${ }^{5}$ siendo en muchas ocasiones el único factor encontrado en relación al ictus isquémico en adultos jóvenes sin factores de riesgo cardiovascular.

Existe tres teorías de la relación del FOP con el ictus: la embolización paradójica, con la formación de trombos en el sistema venoso periférico que 
pasan a través del defecto interauricular; la formación in situ de trombos, y la presencia de arritmias favorecidas por los cambios estructurales, siendo la primera de ellas la más aceptada. ${ }^{3}$ Sin embargo, la simple presencia del FOP no es el único condicionante para un ACVI, y este suele estar asociado a factores de hipercoagulabilidad, trastornos que incrementan la presión en el cavidades cardiacas derechas (hipertensión pulmonar, tromboembolia pulmonar, insuficiencia tricuspídea) y otros, ${ }^{4}$ pues no existe evidencia que demuestre mayor riesgo respecto a quienes no tienen FOP. ${ }^{6}$

La sospecha clínica debe tenerse en adultos jóvenes (generalmente, $<50$ años), un evento posterior a una maniobra de valsalva, ausencia de otros factores de riesgo cardiovascular y la localización del infarto en corteza cerebral (siendo menos probable el embolismo en infartos profundos).

Para hacer el diagnóstico de FOP en la paciente, se utilizó el estándar de oro, que es la visualización del defecto mediante ecocardiografía transesofágica (ETE) con la visualización de solución aireada a través del FOP, también conocida como "prueba de burbujas", la cual cuenta con una sensibilidad y especificidad cercana al 100\%. ${ }^{7}$ A pesar de ser el ecocardiograma transtorácico el estudio más utilizado, no se recomienda para fines de tamizaje por su baja sensibilidad (50-60\%). ${ }^{4}$

Existen factores asociados a un mayor riesgo de ACVI en pacientes con FOP, como la presencia de aneurisma septal atrial $(>10 \mathrm{~mm})$, la hipermovilidad septal, y el tamaño del cortocircuito, siendo considerado grande cuando se observa el paso de más de 30 microburbujas en los primeros tres latidos cardiacos en el ETE; ${ }^{8}$ mismas características que presenta nuestra paciente.

A pesar de ser bajo el riesgo de recurrencia (1.16\%), ${ }^{9}$ los últimos ensayos clínicos realizados en pacientes con FOP han demostrado superioridad del cierre percutáneo contra el tratamiento médico, incluyendo antiagregación, anticoagulación o ambas, ${ }^{10,11,12}$ respaldado por metanálisis con una disminución significativa del riesgo. ${ }^{9}, 13,14,15,16$

Cabe resaltar que existe un riesgo incrementado de presentar fibrilación auricular en los pacientes que se someten a cierre percutáneo del defecto, que pareciera estar relacionado mayormente con el tipo de dispositivo utilizado, apareciendo en los primeros 45 días; por lo que el seguimiento electrocardiográfico en estos pacientes es fundamental. Sin embargo, se requieren estudios de seguimiento a largo plazo para determinar el impacto clínico de esta complicación, dado que, hasta el momento, la evidencia apunta a un mayor beneficio en el cierre del FOP en pacientes con ACVI de origen criptogénico. ${ }^{17}$

En nuestro país se han realizado diversos estudios que señalan la importancia de clasificar adecuadamente el FOP para definir el plan adecuado a seguir, según su clasificación en FOP de alto riesgo o no, ya que, si es de alto riesgo, se debe ofrecer de primera instancia corrección quirúrgica. Se determina de alto riesgo cuando: se presenta neurisma septal atrial (con excursión $>10 \mathrm{~mm}$ ) y el tamaño del forámen es $>2 \mathrm{~mm} .{ }^{18,19}$

A lo anterior se agrega la clara necesidad del Doppler en el ecocardiograma como herramienta diagnóstica en foramen oval permeable como causa de evento vascular cerebral de presunto origen criptogénico. Por lo que sugerimos al lector que, dentro de sus abordajes, no deje de lado dicho procedimiento. 


\section{Referencias}

1. Béjot Y, Bailly H, Durier J, Giroud M. Epidemiology of stroke in Europe and trends for the 21st century. Presse Med 2016; 45:e391-98.

2. Merel S, Esther M, Aneesh B, Kay Sin Tan, Stephanie Debette, Anil M. Epidemiology, aetiology, and management of ischaemic stroke in young adults. Lancet Neurol 2018; 17:790-801.

3. Miranda B, Fonseca AC, Ferro JM. Patent foramen ovale and stroke. J Neurol. 2018; 265(8):1943-1949. doi:10.1007/s00415-018-8865-0

4. Homma S, Messé SR, Rundek T, et al. Patent foramen ovale. Nat Rev Dis Primers. 2016; 2:15086. Publicado 21 Ene 2016. doi: 10.1038/nrdp.2015.86

5. Meissner I, Whisnant JP, Khandheria BK, et al. Prevalence of potential risk factors for stroke assessed by transesophageal echocardiography and carotid ultrasonography: the SPARC study. Stroke Prevention: Assessment of Risk in a Community. Mayo Clin Proc. 1999; 74(9):862-869. doi: 10.4065/74.9.862

6. Serena J, Jiménez-Nieto M, Silva $Y$, Castellanos M. Patent foramen ovale in cerebral infarction. Curr Cardiol Rev. 2010; 6(3):162-174. doi: $10.2174 / 157340310791658794$

7. Sun YP, Homma S. Patent Foramen Ovale and Stroke. Circ J. 2016; 80(8):1665-1673. doi: 10.1253/circj.CJ-16-0534

8. Turc G, Lee JY, Brochet E, et al. Atrial Septal Aneurysm, Shunt Size, and Recurrent Stroke Risk in Patients With Patent Foramen Ovale. J Am Coll Cardiol. 2020; 75(18):2312-2320. doi: 10.1016/j.jacc.2020.02.068

9. Ahmad Y, Howard JP, Arnold A, et al. Patent foramen ovale closure vs. medical therapy for cryptogenic stroke: a meta-analysis of randomized controlled trials. Eur Heart J. 2018; 39(18):1638-1649. doi: 10.1093/eurheartj/ehy121

10. Mas JL, Derumeaux G, Guillon B, et al. Patent Foramen Ovale Closure or Anticoagulation vs. Antiplatelets after Stroke. N Engl J Med. 2017; 377(11):1011-1021. doi: 10.1056/NEJMoa1705915

11. Søndergaard L, Kasner SE, Rhodes JF, et al. Patent Foramen Ovale Closure or Antiplatelet Therapy for Cryptogenic Stroke [published correction appears in N Engl J Med. 5 Mar 2020; 382(10):978]. N Engl J Med. 2017; 377(11):1033-1042. doi: 10.1056/NEJMoa1707404

12. Lee PH, Song JK, Kim JS, et al. Cryptogenic Stroke and High-Risk Patent Foramen Ovale: The DEFENSE-PFO Trial. J Am Coll Cardiol. 2018; 71(20):2335-2342. doi: 10.1016/j.jacc.2018.02.046

13. Ntaios G, Papavasileiou V, Sagris D, et al. Closure of Patent Foramen Ovale Versus Medical Therapy in Patients With Cryptogenic Stroke or Transient Ischemic Attack: Updated Systematic Review and Meta-Analysis. Stroke. 2018; 49(2):412-418. doi: 10.1161/STROKEAHA.117.020030

14. Shah R, Nayyar M, Jovin IS, et al. Device Closure Versus Medical Therapy Alone for Patent Foramen Ovale in Patients With Cryptogenic Stroke: A Systematic Review and Meta-analysis [published correction appears in Ann Intern Med. 18 Sep 2018; 169(6):428]. Ann Intern Med. 2018; 168(5):335-342. doi: 10.7326/ M17-2679

15. Schulze V, Lin Y, Karathanos A, et al. Patent foramen ovale closure or medical therapy for cryptogenic ischemic stroke: an updated meta-analysis of randomized controlled trials. Clin Res Cardiol. 2018; 107(9):745-755. doi: 10.1007/ s00392-018-1224-4 
16. De Rosa S, Sievert H, Sabatino J, Polimeni A, Sorrentino S, Indolfi C. Percutaneous Closure Versus Medical Treatment in Stroke Patients With Patent Foramen Ovale: A Systematic Review and Meta-analysis. Ann Intern Med. 2018; 168(5):343-350. doi: 10.7326/M17-3033

17. Mojadidi MK, Zaman MO, Elgendy IY, et al. Cryptogenic Stroke and Patent Foramen Ovale. J Am Coll Cardiol. 2018; 71(9):1035-1043. doi: 10.1016/ j.jacc.2017.12.059

18. Cruz-GI, Solisa J, Inglessis AI, et al. Foramen oval permeable: situación actual Patent Foramen Ovale: Current State of the Art, Revista Española de Cardiología, Jul 2008; 738-751. doi: 10.1157/13123995.

19. González-PMA, Mena JE, Rodríguez FG, Foramen oval permeable asociado con enfermedad vascular cerebral de tipo isquémico; controversia en su tratamiento. Experiencia en el Hospital Ángeles Pedregal Med Int Méx 2014; 30:511-519. 